\title{
Tuning the Separation Properties of Zeolitic Imidazolate Framework Core-Shell Structures via Post-Synthetic Modification
}

Javier Sánchez-Laínez ${ }^{\mathrm{a}}$, Adrián Veiga ${ }^{\mathrm{a}}$, Beatriz Zornoza ${ }^{\mathrm{*}}$, Salvador R.G. Balestrab ${ }^{\mathrm{b}}$, Said Hamad ${ }^{\mathrm{b}}$, A. Rabdel Ruiz-Salvador ${ }^{b}$, Sofia Calero ${ }^{b}$, Carlos Téllez $^{\mathrm{a}}$ and Joaquín Coronas ${ }^{\mathrm{a} *}$

The conversion of ZIF-8 into ZIF-7 via post-synthetic modification with benzimidazole has been monitored quantifying the liberated 2-methylimidazole by chromatography. The reaction kinetics has been adjusted to the shrinking core model, providing the diffusion coefficient of blm inside the pores and the reaction kinetic constant $(2.86 \cdot 10-7 \mathrm{~cm} 2 / \mathrm{s}$ and $1.36 \cdot 10-4 \mathrm{~cm} / \mathrm{s}$, respectively). A wide variety of ZIF-7/8 hybrid core-shell frameworks has been obtained during this reaction. The most promising have been characterized by SEM/TEM, TGA, N2 and CO2 adsorption, FTIR and 13C NMR, showing features of the coexistence of both phases inside the frameworks. Their structures have also been simulated, providing comparable XRD and adsorption results. The hybrid material has been used as filler for PBI mixed matrix membranes (MMMs) applied to $\mathrm{H} 2 / \mathrm{CO} 2$ separation, enhancing the performances of the bare PBI polymer and MMMs containing ZIF-8 or ZIF-7 as fillers, with a maximum value of 1921 Barrer of H2 and a $\mathrm{H} 2 / \mathrm{CO} 2$ selectivity of 11.8 .

\section{1- Introduction}

Zeolitic Imidazolate Frameworks (ZIFs) are a subfamily of metal-organic frameworks (MOFs), where $\mathrm{Zn2}+$ or $\mathrm{Co} 2+$ cations in tetrahedral coordination are covalent bounded to organic linkers based on imidazole groups. In these frameworks, metal-imidazole-metal angles are close to $145^{\circ}$, which is coincident with the Si-O-Si angles typically found in zeolites. ZIFs constitute highly porous frameworks with extraordinarily high thermal and chemical stabilities and they have a great number of potential applications such as gas sorption and separation,1-3 drug delivery4 and catalysis.5,6 Since first discovered independently by Yaghi's7 and Chen's groups, 8 the number and variety of ZIFs has increased in the last few years, with a significant use of a huge number of organic linkers. 9 The combination of different linkers inside the same crystalline structure has appeared as a novel route to develop new hybrid materials that can combine the beneficial properties of their original frameworks. Mixtures of linkers have already been reported for the direct solvothermal synthesis of hybrid ZIFs, such as ZIF-7-9010, 11, ZIF-7-811 and ZIF-9-67.12 In addition, the series ZIF-68 to ZIF-70,13 ZIF-78 to ZIF-8214 and ZIF-300 to ZIF-30215 constitute hybrid ZIFs with a mixture of linkers in their structure. However, some of the challenges of direct synthesis are limited linker solubility, chemical and thermal stability, functional group compatibility and particle size control. These problems may be avoided employing post-synthetic modification routes. In this manner, materials of high complexity and functionality can be obtained avoiding MOF destruction during the chemical reaction. This methodology has been successfully applied to a large number of MOFs,16 including the aldehyde groups in ZIF-9017 and SIM-1.18 Special ZIF/ZIF hybrid structures named core-shell structures can also be obtained as reported for ZIF- 8 by Liu et al. 19 and Nair et al.20 
The pore architecture is a key element for gas separation of the $\mathrm{H} 2 / \mathrm{CO} 2$ mixture, typically obtained at high pressure and temperature during hydrogen production via the steam reforming of methane. Two interesting frameworks for this gas separation are ZIF-8 and ZIF-7, both sharing sod topology, built by 2-methylimidazole $(\mathrm{mlm})$ and benzimidazole $(\mathrm{blm})$, respectively. ZIF-8 crystallizes in cubic symmetry, but the large size of blm renders crystallization of ZIF-7 in rhombohedral symmetry, also featuring large ligand rotations. Despite the large size difference of the ligands, these ZIFs show relatively similar pore apertures of 0.34 and $0.30 \mathrm{~nm}$, respectively, close to the kinetic diameter of $\mathrm{H} 2(0.29 \mathrm{~nm})$, making them ideal for the separation of this gas over larger molecules such as $\mathrm{CO} 2(0.33 \mathrm{~nm})$ and $\mathrm{CH} 4(0.38 \mathrm{~nm})$. The use of polybenzimidazole (PBI) membranes for the separation of $\mathrm{H} 2 / \mathrm{CO} 2$ mixtures has been widely reported 21-26 and several types of ZIFs, such as ZIF-722, ZIF-827-29, ZIF-1123, 30 and ZIF-9031, have been embedded in PBI to create mixed matrix membranes (MMMs). Despite the smaller pore windows of ZIF-7 and ZIF-11, compared to those of ZIF-8, they showed worse gas separation performance when embedded in PBI.22, 30 A core-shell hybrid material of ZIF-7 and ZIF-8 is expected to result in a ZIF with enhanced separation performance, owing to its better compatibility with the polymer provided by the blm linker from ZIF-7 (shell) and the reduced $\mathrm{CO} 2$ adsorption and good $\mathrm{H} 2$ diffusivity of ZIF-8 (core).

This work presents the synthesis of hybrid ZIFs with a core-shell configuration, showing the tunable nature of this kind of materials. ZIF-7/8 core-shells were obtained through the postsynthetic modification of ZIF-8, reaction that was monitored and adjusted to the shrinking core model. This kinetic model let define with high accuracy the reaction conditions to obtain a great variety of hybrid frameworks with different compositions. The material can be therefore adapted to certain necessities according to its final application. In this case of study, incorporating just a $10 \%$ of ZIF-7 to ZIF-8 allowed an enhanced separation performance for $\mathrm{H} 2 / \mathrm{CO} 2$ separation (CO2 pre-combustion capture) when embedded in PBI MMMs owing to: (a) the better filler-polymer compatibility provided by benzimidazole ligand from ZIF-7, acting as shell, and (b) the reduced $\mathrm{CO} 2$ adsorption and high $\mathrm{H} 2$ diffusivity of ZIF-8, acting as core. The concepts of core-shell and reaction monitoring may be extended to other MOFs and different applications, so that the frameworks can modify their properties to enhance their performance.

\section{2- Experimental Section}

\subsection{Chemicals:}

Zinc nitrate hexahydrate $(\mathrm{Zn}(\mathrm{NO} 3) 2 \cdot 6 \mathrm{H} 2 \mathrm{O}), 2$ 2-methylimidazole $(\mathrm{mlm}, \mathrm{C} 4 \mathrm{H} 6 \mathrm{N2},>99 \%)$, benzimidazole (blm, C7H6N2, 98\%) and N,N-Dimethylacetamide (DMAc, $\geq 99.5 \%$ ), were purchased from Sigma Aldrich. Methanol (MeOH, HPLC grade) and N,N-Dimethylformamide (DMF, synthesis grade) were purchased from Scharlau. Commercial PBI solution comprising 26 
wt\% PBI with $1.5 \mathrm{wt} \% \mathrm{LiCl}$ as stabilizer in DMAc solvent was purchased from PBI Performance Products.

\subsection{MOFs synthesis:}

ZIF-8 was synthesized according to the following procedure based on the literature. $32470 \mathrm{mg}$ of $\mathrm{Zn}$ (NO3)2.6H2O was dissolved in $10 \mathrm{~mL}$ of MeOH and $10 \mathrm{~mL}$ of water. Besides, $1.0 \mathrm{~g}$ of mlm was dissolved in $10 \mathrm{~mL}$ of $\mathrm{MeOH}$, and the two solutions were mixed and stirred for $2 \mathrm{~h}$. The final product was collected by centrifugation, washed once with $\mathrm{MeOH}$, and dried at $110{ }^{\circ} \mathrm{C}$ overnight. The resulting nanoparticles had a particle size of about $150 \mathrm{~nm}$.

ZIF-7 was also synthesized in order to compare the effects caused on ZIF-8 framework after its post-synthetic modification. The following receipt found in the literature was followed.33 1.63 $\mathrm{g}$ of blm and $0.64 \mathrm{~g}$ of $\mathrm{Zn}(\mathrm{NO} 3) 2 \cdot 6 \mathrm{H} 2 \mathrm{O}$ were dissolved in $200 \mathrm{~mL}$ of DMF. The solution was stirred at room temperature for $48 \mathrm{~h}$ and the solid was collected by centrifugation. Afterthat the solid was activated in $\mathrm{MeOH}$ at $60^{\circ} \mathrm{C}$ under reflux for $18 \mathrm{~h}$.

The ZIF-7/8 core-shells were prepared by post-synthetic modification of ZIF-8. An initial amount of blm was added to DMF at $65{ }^{\circ} \mathrm{C}$ to obtain a concentration of $13 \mathrm{~g} / \mathrm{L}$. When solved, ZIF-8 was added to the solution ( $3.3 \mathrm{~g} / \mathrm{L})$; which was stirred. The solid was collected at the corresponding reaction time by centrifugation and washed 3 times with $\mathrm{MeOH}$ and dried at $110^{\circ} \mathrm{C}$ overnight. The $\mathrm{m} / \mathrm{m} / \mathrm{b} / \mathrm{m}$ proportion in the ZIF-7/8 hybrid was calculated assuming that all the liberated $\mathrm{mlm}$ had been replaced by blm during the reaction. Different initial blm concentrations and temperatures were also tested to study their influence on the reaction time (see Table S1).

\subsection{Kinetic study:}

The kinetic study of the post-synthetic modificaction reaction of ZIF-8 was performed quantifying the amount of $\mathrm{mlm}$ liberated by Gas Chromatography/Mass Spectrometry GC/MS, taking aliquots during the reaction time. The ZIF-8 conversion was calculated as the amount of $\mathrm{mIm}$ quantified in the reaction medium divided by the total amount present in the original ZIF8 structure. The equipment used was an Agilent $6850 \mathrm{GC}$ system with a capillary column HP5MS $(30 \mathrm{~m} \times 0.250 \mathrm{~mm} \times 0.25 \mu \mathrm{m})$ coupled with an Agilent $5975 \mathrm{MSD}$. The detector and injector temperature were $200{ }^{\circ} \mathrm{C}$ and helium was used as the carrier gas. The column was set at $110^{\circ} \mathrm{C}$. An aliquot of $1.5 \mathrm{~mL}$ was taken from the reaction medium after the reaction had finished and it was injected at least three times to get the average concentration.

\subsection{MMM preparation:}

The required amount of ZIF (ZIF-7/8 core-shell, neat ZIF-7 and neat ZIF-8) was weighed for each membrane loading, from 10 to $40 \mathrm{wt} \%$, and the corresponding amount of PBI solution was added (previously diluted in DMAc to $15 \mathrm{wt} \%$ concentration to avoid problems deriving 
from viscosity). The resulting solution was further stirred overnight. The casting solution was three times stirred and sonicated alternately for $90 \mathrm{~min}$ total time and cast into a Petri dish leveled inside an oven at $90{ }^{\circ} \mathrm{C}$. The petri dishes were left uncovered overnight to allow the evaporation of the solvent. After that, the membranes were peeled off from the petri dishes and washed for $24 \mathrm{~h}$ in $\mathrm{MeOH}$. Finally, the membranes were treated in an oven at $100{ }^{\circ} \mathrm{C}$ and for $24 \mathrm{~h}$ for complete removal of the remaining solvent. The membrane thicknesses were tested by a Digimatic Micrometer (measurement range from 0 to $30 \mathrm{~mm}$ with an accuracy of $\pm 1 \mu \mathrm{m}$ ). Several equally distributed points (9) on each membrane were measured and the arithmetic mean was taken as the membrane thickness. The MMMs obtained in this work had a thickness of $88 \pm 16 \mu \mathrm{m}$. For permeation testing of the membranes, circular areas of $3.14 \mathrm{~cm} 2$ were cut from the films.

\subsection{Characterization of samples:}

Powder X-ray diffraction (XRD) spectra of the MOFs and MMMs were acquired using a D-Max Rigaku X-ray diffractometer with a copper anode and a graphite monochromator to select CuK $\alpha$ radiation $\left(\lambda=1.540 \AA\right.$ ) , taking data from $2 \theta=2.5^{\circ}$ to $40^{\circ}$ at a scan rate of $0.03 \%$. Thermogravimetric analyses (TGA) were carried out using a Mettler Toledo TGA/STDA 851e. Samples $\left(5 \mathrm{mg}\right.$ ) placed in $70 \mathrm{LL}$ alumina pans were heated in air flow from 30 to $900{ }^{\circ} \mathrm{C}$ at a heating rate of $10{ }^{\circ} \mathrm{C} / \mathrm{min}$. Scanning electron microscopy (SEM) images of the ZIFs and membranes were obtained using an Inspect F50 model scanning electron microscope (FEI), operated at $20 \mathrm{kV}$. Cross-sections of membranes were prepared by freeze-fracturing after immersion in liquid nitrogen and subsequent coating with Pt. Transmission electron microscopy (TEM, FEI TECNAI F30) images of the ZIF samples were acquired at an acceleration voltage of $300 \mathrm{~kW}$. This TEM, fitted with a SuperTwin ${ }^{\circledR}$ lens allowing a point resolution of $1.9 \AA$, is equipped for spectroscopy experiments performed in EDS (X-Ray Microanalysis). Samples were prepared by placing one drop of a dilute suspension of powder in $\mathrm{MeOH}$ on a holey carbon-coated copper grid (300 mesh) and allowing the solvent to evaporate at room temperature. Particle size was obtained using ImageJ $1.49 \mathrm{~b}$ software, where at least 60 particles were counted for each sample. Fourier transform infrared spectroscopy (FTIR) was performed on a Bruker Vertex 70 FTIR spectrometer equipped with a DTGS detector and a Golden Gate diamond ATR accessory. Both spectra were recorded by averaging 40 scans in the 4000-600 cm-1 wavenumber range at a resolution of $4 \mathrm{~cm}-1$. Nitrogen adsorption/desorption isotherms were obtained using a Micromeritics Tristar 3000 surface area and porosity analyzer, after previously degassing the samples both powders and MMM at $200{ }^{\circ} \mathrm{C}$ for $8 \mathrm{~h}$ under vacuum. NMR Spectra were recorded in a Bruker Avance III WB400 spectrometer with 4 $\mathrm{mm}$ zirconia rotors spun at the magic angle in $\mathrm{N} 2$ at $10 \mathrm{kHz}$. $1 \mathrm{H}-13 \mathrm{C} \mathrm{CP}$ spectra were measured using a $1 \mathrm{H} \pi / 2$ pulse length of $3.0 \mu \mathrm{s}$, with a contact time (ramp) of $3 \mathrm{~ms}$, a spinal 64 proton decoupling sequence of $5.3 \mu \mathrm{s}$ pulse length, and a recycle delay of $5 \mathrm{~s} .3000$ scans were acquired for each spectrum. The chemical shifts were reported relative to TMS.

\subsection{Gas separation analysis:}

Mixed gas analyses were performed for PBI MMMs containing 10, 20 and $32 \mathrm{wt} \%$ loading of the ZIF-7/8 core-shell. The results were compared to those of neat PBI membranes and PBI 
based MMMs with ZIF-8 and ZIF-7. The membranes were placed in a module consisting of two stainless steel pieces and a 316LSS macroporous disk support (from Mott Co.) with a $20 \mu \mathrm{m}$ nominal pore size, and gripped inside with Viton O-rings. The permeation module was placed in a UNE 200 Memmert oven to control the temperature of the experiments. Gas separation measurements were carried out by feeding a $\mathrm{H} 2 / \mathrm{CO} 2$ equimolar mixture (25/25 $\mathrm{cm} 3(\mathrm{STP}) / \mathrm{min}$ ) at 3 bar to the feed side by means of two mass-flow controllers (Alicat Scientific, MC-100CCM-D), while the permeate side of the membrane was swept with a 2 $\mathrm{cm} 3(\mathrm{STP}) / \mathrm{min}$ mass-flow controller stream of Ar at 1 bar (Alicat Scientific, MC-5CCM-D). Concentrations of $\mathrm{H} 2$ and $\mathrm{CO} 2$ in the outgoing streams were analyzed by an Agilent 3000A online gas microchromatograph equipped with a thermal conductivity detector (TCD). Permeability was calculated in Barrer $(10-10 \mathrm{~cm} 3($ STP $) \cdot \mathrm{cm} /(\mathrm{cm} 2 \cdot \mathrm{s} \cdot \mathrm{cmHg}))$ once the steadystate of the exit stream was reached (for at least $3 \mathrm{~h}$ ), and the separation selectivity was calculated as the ratio of permeabilities. At least 2-3 MMM samples of each loading were fabricated and measured to provide the corresponding error estimations.

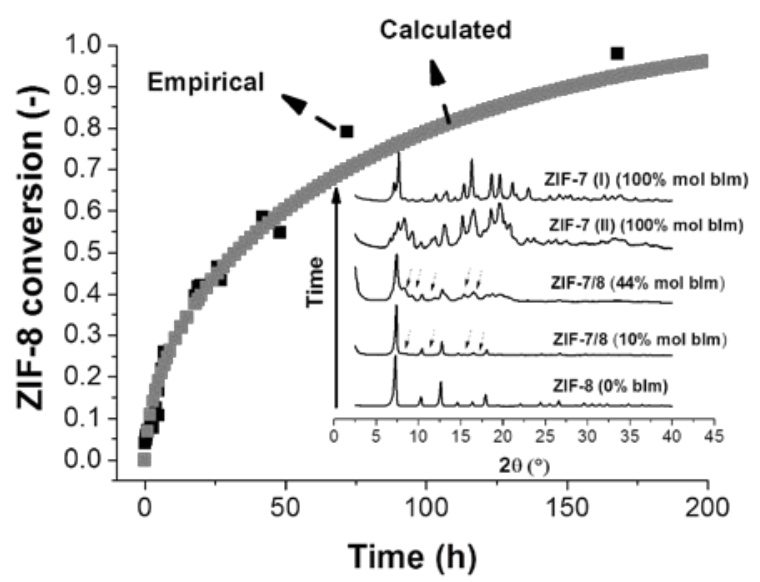

Fig. 1. Conversion of ZIF-8 vs. time at $65{ }^{\circ} \mathrm{C}$ : empirical (black) and calculated (grey) values with the shrinking core model.

\subsection{Simulation:}

Simulations were performed with RASPA34. Adsorption isotherms of $\mathrm{CO} 2$ and $\mathrm{H} 2$ at $273 \mathrm{~K}$ were simulated using Grand Canonical MonteCarlo (GCMC, $\mu \mathrm{VT}$ ) simulations, using 250000 steps and the Configuration Bias MC algorithm in the insertion/deletion moves. The isosteric heats of adsorption of the guest molecules were computed after 200000 sampling steps using the Widom Insertion Method. Host-guest interactions were modeled with Lennard-Jones (LJ) pairwise interatomic potentials plus electrostatic interactions. The atomic charges were taken from comuted values by Gutierrez-Sevillano et al.35 The values of the $L J$ parameters used in the simulations were obtained through Lorentz-Berthelot mixing rules, for which the force field parameters of the atom of the MOF were taken from the UFF force field for $\mathrm{Zn}$ atom and Dreding force field for the ligands.36, 37 Diffusion coefficients were calculated through the linear fit of the Mean Square Displacement (MSD) of the guest in the infinite dilution regime at $453 \mathrm{~K}$ and $523 \mathrm{~K}$. The MSD were simulated using Molecular Dynamics simulation in the canonical ensemble (NVT MD),38 using a time step of $0.5 \mathrm{fs}$ and 250 and $1000 \mathrm{ps}$ of 
equilibration and production, respectively. Structures were always optimized to guaranteed realistic adsorption and transport properties. The force field developed by Wu was used for the geometry optimization of the materials.39

\section{3- $\quad$ Results and discussion}

\subsection{Reaction kinetics}

To deduce the reaction kinetics, the reaction was monitored measuring by GC/MS the liberation of $\mathrm{mIm}$ from ZIF-8. The data obtained were fitted to the shrinking core model for spherical particles of unchanging size proposed by Levenspiel.40 This model for fluid-solid reaction considers that the reaction occurs first at the outer skin of the particle. The zone of reaction then moves into the solid, leaving behind completely converted material and inert solid (ash). We consider in our reaction the original ZIF-8 nanoparticles as the solid phase and the blm (ZIF-7 benzimidazolate ligand) solution as the liquid phase. The converted material would be the ZIF-7, formed in the shell of the hybrid structure during the post-synthetic modification. The film resistance at the surface of the particle was neglected, simplifying the model to the expression shown in Eq. (S1). To the best of our knowledge, this is the first time that this reaction in MOFs has been studied by means of a kinetic model.

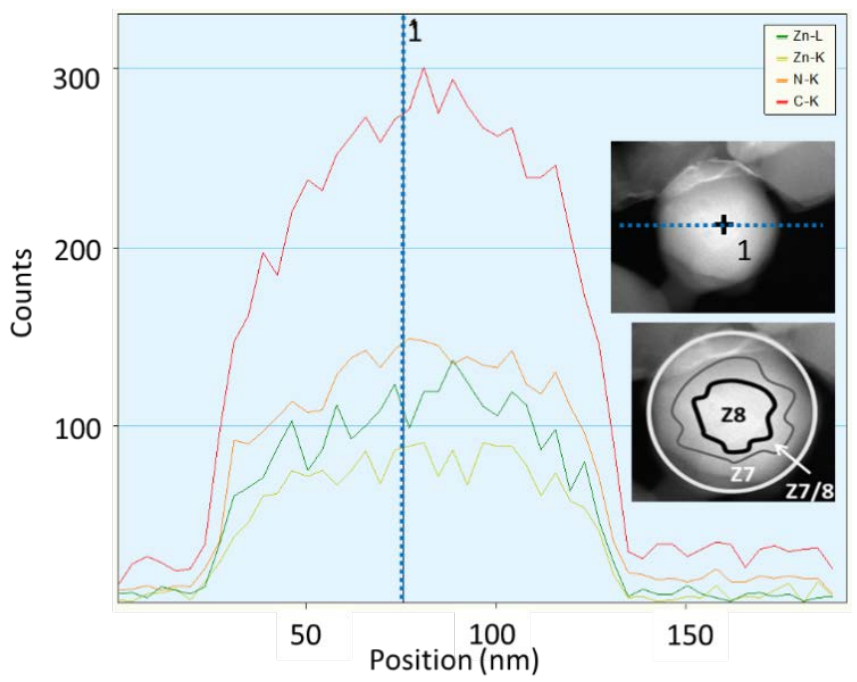

Fig. 2. EDX profile of the single $10 \% \mathrm{~mol}$ blm hybrid nanoparticle in the top inset. From up to down: $\mathrm{C}-\mathrm{K}, \mathrm{N}-\mathrm{K}, \mathrm{Zn}-\mathrm{L}$ and $\mathrm{Zn}-\mathrm{K}$. The bottom inset corresponds to a scheme of this particle.

The analysis of the exchange reaction reveals that the diffusion inside the pores appears to be the reaction controlling step, since it is responsible for around the $90 \%$ of the total reaction time. The diffusion coefficient at the experimental temperature $\left(65^{\circ} \mathrm{C}\right), 2.86 \cdot 10-7 \mathrm{~cm} 2 \cdot \mathrm{s}-1$, as extracted from the fitting of the conversion curve (Fig. 1 and Table S2), indicates relatively large diffusivity of the ligands through the sod-type ZIFs. This is surprising since the kinetic diameter of blm is at least that of benzene $(0.585 \mathrm{~nm})$ and therefore this is new evidence of the extremely high flexibility of this framework. Although it has been previously reported how 
long guest molecules can diffuse as quickly as short molecules in ZIF-8.41 The calculated kinetic constant was $1.36 \cdot 10-4 \mathrm{~cm} \cdot \mathrm{s}-1$. The quality of the fitting of the experimental data to the kinetic model is good (see \% error in Table S3), with relatively larger errors (up to $20 \%$ ) for the initial data at reaction times up to $5 \mathrm{~h}$. This may be due to a different mechanism at the beginning of the reaction, probably related to desorption and partial dissolution processes, which may also explain the traces of $\mathrm{Zn}$ found in the reaction medium (Fig. S1).

A full range of hybrid materials was obtained until the complete transformation of ZIF-8 into ZIF-7 occurred, showing intermediately the structure of a narrow pore ZIF-742. This progression can be seen in the diffraction patterns of Fig. 1(inset), indicating that a shrinking mechanism controls the process. The hybrid framework maintains the cubic structure of ZIF-8, while additional peaks can be observed at $2 \theta=8.4,9.4,11.8,15.2$ and $16.6^{\circ}$, belonging to the ZIF-7(II) pattern.42 Given that the contribution of bIm to the diffraction pattern of the ZIF-7/8 hybrid is as crystalline ZIF-7, its incorporation must have occurred by linker exchange with $\mathrm{mIm}$ during the post-modification reaction. Otherwise its interaction with the $\mathrm{Zn} 2+$ cations of ZIF-8 to build the ZIF-7 structure would have been impossible.

\subsection{Core-shells characterization}

Two core-shells with different amounts of blm (10 and 44 mol\%) were chosen for further characterization, the former being the first with visible ZIF-7 XRD reflections and the latter, having similar quantities of each linker. Note that ZIF-8 and ZIF-7(II) phases are clearly identified from the pattern of ZIF-7/8 (44\% blm) in Fig. 1 (inset). The large peak width supports this view, which can be interpreted as a consequence of the small particle size corresponding to each phase. The simulated XRD shows how the diffractogram of the hybrid material (Fig. S2) fits better a linear combination of neat ZIF-7 and ZIF-8 patterns than the corresponding XRD pattern of a homogeneous mixed-ligand structure at cell unit level, suggesting the existence of a core-shell arrangement of phases.

Regarding the particles' morphology, a single particle size population or morphology can be observed in the SEM/TEM images (Fig. S3), consistent with the existence of one kind of particle, instead of a physical mixture of ZIF-7 and ZIF-8. The particle size decreased from 150 $\mathrm{nm}$ of the original ZIF-8 to $124 \mathrm{~nm}$, according to the cumulative distribution graph shown in Fig. S4 and seems to be related to the partial dissolution of $\mathrm{Zn}$ found previously. EDX analysis performed on a single nanoparticle, revealed that the $\mathrm{Zn}$ and $\mathrm{N}$ amounts remain constant across the particle width (Fig. 2), with a consistent proportion in the framework structure (Table S4). 


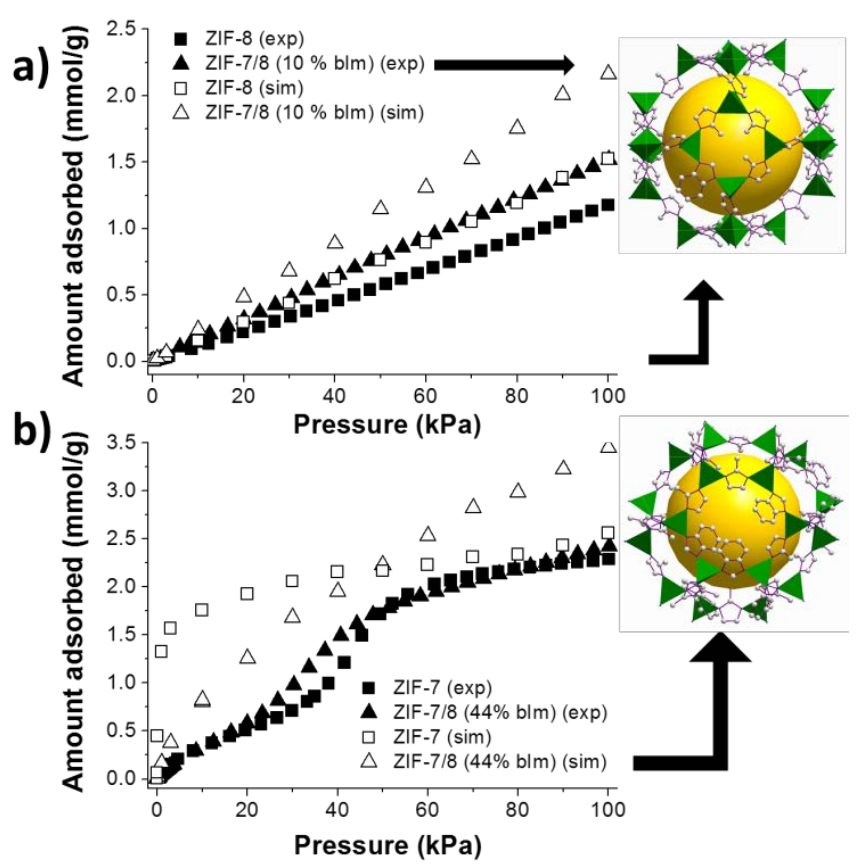

Fig. 3. Simulated and empirical $\mathrm{CO}_{2}$ adsorption isotherms of ZIF-7 (a), ZIF-8 (b) and both hybrid materials (a, b) at $273 \mathrm{~K}$ and simulated structure (inset).

The thermal stability of the core-shells was also tested. The TGA of the hybrid material in air (Fig. S5) shows an intermediate behavior between ZIF-7 and ZIF-8, with two decomposition steps corresponding to the coexistence of $\mathrm{mlm}$ and blm ligands in the structure. The onset temperatures are slightly different to those of the neat ZIFs ( 472 vs. $455^{\circ} \mathrm{C}$ for $\mathrm{mIm}$ and 527 vs. $576{ }^{\circ} \mathrm{C}$ for blm). The $\mathrm{mIm}$ in the hybrid structure is more stable than that in ZIF-8, but the opposite happens to blm linker. This may be due to the fact that blm is more exposed, as it is mainly located at the outer part of the framework, while $\mathrm{mlm}$ is more protected, basically remaining in the inner part.

The adsorption properties of the core-shells were also tested to study their microporosity in deep. The two $10 \%$ and $44 \%$ ZIF-7/8 hybrids show N2 uptakes of 374 and $290 \mathrm{~cm} 3(\mathrm{STP}) / \mathrm{g}$ at $\mathrm{P} / \mathrm{PO}=1$ (Fig. S6), about one fourth and one half smaller than that of ZIF-8, respectively. The same occurs with the BET area, which is $20 \%$ smaller for the hybrid with the lowest amount of blm $(1050 \mathrm{~m} 2 / \mathrm{g})$. This reduction is even higher for the other hybrid, having a value of 719 $\mathrm{m} 2 / \mathrm{g}$. The inclusion of blm in the ZIF-8 framework structure (in the form of ZIF-7 according to the XRD spectra) reduces the maximum quantity adsorbed in comparison to the original ZIF-8. This causes a partial reduction of the pore windows in the hybrid material, hindering the entrance of N2 molecules inside the framework structure (in fact, ZIF-7 does not adsorb N2).43 Our adsorption measurement suggests that the shell part of our hybrid material is expected to have a small amount of the parent $\mathrm{m} / \mathrm{m}$ ligand that create percolative paths to the diffusion of $\mathrm{N} 2$ in terms of the adsorption capacity. 


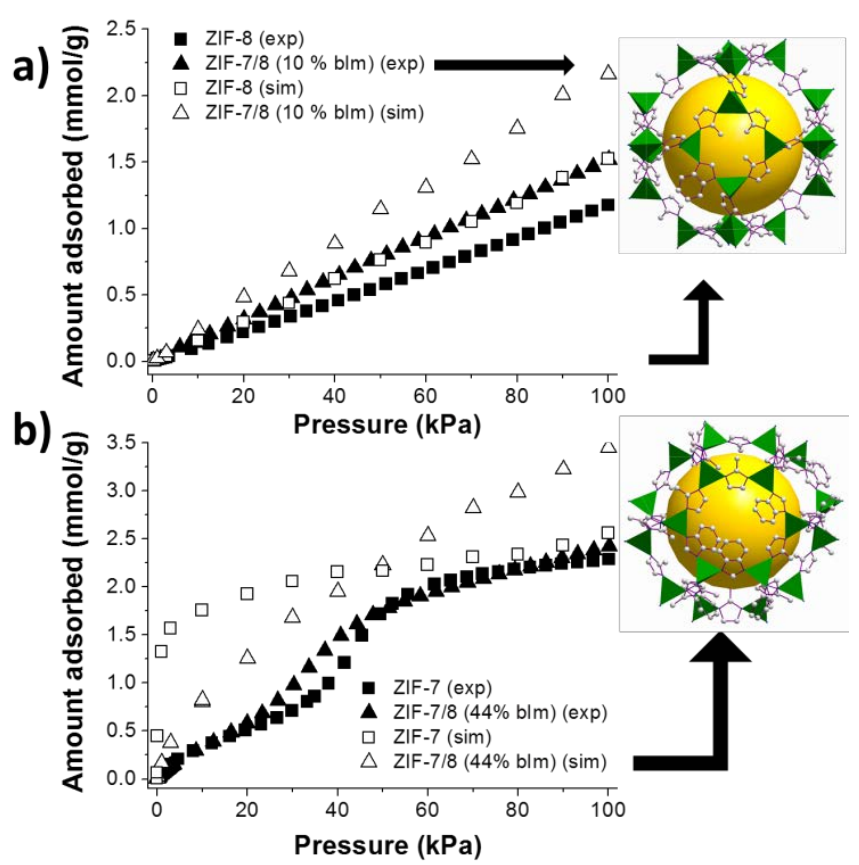

Fig. 4. Simulated and empirical $\mathrm{CO}_{2}$ adsorption isotherms of ZIF-7 (a), ZIF-8 (b) and both hybrid materials (a, b) at $273 \mathrm{~K}$ and simulated structure (inset).

The opposite tendency can be seen for $\mathrm{CO} 2$ adsorption (Fig. 3 and S7) since ZIF-8 is the framework with the lowest adsorption capacity. It is worth noting that the hybrid containing $44 \%$ of blm adsorbs the same quantity of $\mathrm{CO} 2$ as the bare ZIF-7 despite containing half the amount of linker. The other hybrid ( $10 \mathrm{wt} \% \mathrm{bIm}$ ), as compared to ZIF-8, adsorbs about $20 \%$ more $\mathrm{CO} 2$, which is consistent with a $90 \%$ core of ZIF-8. The simulated adsorption isotherms shown in Fig. 3 predict well the empirical results. $\mathrm{CO} 2$ and $\mathrm{H} 2$ adsorption heats also increase as the amount of blm in the framework is higher (Table S5).

The chemical composition of the core-shells was studied by $13 \mathrm{C} \mathrm{NMR} \mathrm{(Fig.} \mathrm{4)} \mathrm{and} \mathrm{FTIR} \mathrm{(Fig.} \mathrm{S8).}$ 13C NMR analyses show the resonances of ZIF-8 and ZIF-7 in the hybrids' spectra without any shift, suggesting that a core of ZIF-8 remains unaltered after the post-synthetic modification reaction, and that the chemical environment for the carbon atoms of blm in the hybrid framework is similar to that in neat ZIF-7. 13C NMR spectrum of ZIF-8 (Fig. 4) shows three resonances at $151.2,124.2$ and $13.7 \mathrm{ppm}$ corresponding to the $\mathrm{mlm}$ linker. The former is due to the aromatic carbon linked to nitrogen $(\mathrm{C} 1 \mathrm{~m})$, the second to the $-\mathrm{HC}=\mathrm{CH}-$ group $(\mathrm{C} 2 \mathrm{~m})$ and the latter to the methyl group ( $\mathrm{C} 3 \mathrm{~m}) .44 \mathrm{ZIF}-7$ spectrum also shows the resonances of its linker (blm). The carbon atom between the amino and the imidazole groups (C1b) resonates as a multiplete at $151.0 \mathrm{ppm}$. The signal at $141.7 \mathrm{ppm}$ corresponds to $\mathrm{C} 7 \mathrm{~b}$, while that at 116.2 to $\mathrm{C} 3 \mathrm{~b}, \mathrm{C} 4 \mathrm{~b}$, and C5b resonances. Finally, the peak at 122.9 corresponds to $\mathrm{C} 2 \mathrm{~b}$ and $\mathrm{C} 6 \mathrm{~b}$ resonates at $112.9 \mathrm{ppm} .45-47$

The $13 \mathrm{C}$ NMR experiments also quantified an amount of $18 \% \mathrm{~mol}$ of blm in the framework (see Fig. S9), in relative agreement with the GC-MS (10\%), BET (20\%) and $\mathrm{CO} 2$ adsorption (10\%). The simultaneous validity of diffraction, adsorption and spectroscopy characterizations allows one to depict a ZIF-8-ZIF-7 core-shell particle with a wide hybridized interphase (see scheme in Fig. 2 inset). 


\subsection{Mixed matrix membranes performance}

We considered that the hybrid ZIF could show a good performance as a filler in PBI MMMs for gas separation, especially that with the lowest amount of blm because it retains a good part of the nature of ZIF-8, the ZIF with the best results in the literature, 27-29 while superficially exhibiting a bIm ligand highly compatible with PBI.30 MMMs with loadings from 10 to $32 \mathrm{wt} \%$ (checked by TGA) were prepared and characterized by SEM and XRD. The cross section of the prepared MMMs at different loadings (10, 20 and $32 \mathrm{wt} \%$ ) of ZIF-7/8 hybrid (10 wt\% mol blm) is shown in Fig. S10, where a good interaction between filler and polymer can be clearly distinguished. SEM images were reinforced with EDX analysis (Fig. S11), in which the presence of $\mathrm{Zn}$ from the ZIF nanoparticles can be distinguished across the MMM section. Additionally, XRD spectra of the MMMs (see Fig. S12) show how the filler remains being crystalline once embedded in the polymeric phase. N2 adsorption was also performed on a MMM and the results can be seen in Fig. S13 in comparison to those of a bare PBI membrane and the ZIF-7/8 hybrid core-shell powder. As expected, the MMM shows an intermediate adsorption capacity due to the polymer surrounding the hybrid particles hinders access to N2. In any event, the reduction in the BET area is relatively low as compared to that achieved with ordered mesoporous sphere-polysulfone MMMs where the polymer chains penetrate into the filler porosity.48

PBI MMMs containing ZIF-8 and ZIF-7 were also fabricated and tested under the same conditions for comparison. Fig. 5a shows the $\mathrm{H} 2 / \mathrm{CO} 2$ gas separation performance for a $50 / 50$ mixture at $180^{\circ} \mathrm{C}$ and 3 bar of feed pressure. MMMs with core-shell loadings up to $32 \mathrm{wt} \%$, the maximal loading without the membranes being too brittle, were tested. The integration of the ZIF-7/8 hybrid material improved the membrane performance clearly. When embedding just $10 \mathrm{wt} \%$ of this hybrid filler, the $\mathrm{H} 2$ permeability was seven-fold higher than that of the neat $\mathrm{PBI}$ and the selectivity was doubled. A significant enhancement in the $\mathrm{H} 2$ permeability was observed with increasing loading, reaching a value of $1398 \pm 88$ Barrer at 32 wt\% loading. However, the $\mathrm{H} 2 / \mathrm{CO} 2$ selectivity showed an optimum value of $10.1 \pm 0.2$ at 20 wt\% loading, which is three-fold that of the bare PBI at the same temperature. This suggests the presence of non-selective voids owing to some deterioration in the interaction between the MOF and the polymer at these high loadings. 49 The results evidence the important role that the presence of microporous ZIF-7/8 hybrid plays in the gas transport through the membranes. In the Robeson graph of Fig. 5b, it can be seen how PBI MMMs containing the core-shells have a superior gas separation performance in terms of permeability and selectivity than analogous MMMs with ZIF-7 and ZIF-8 at the same loading, surpassing the Robeson upper bond in all cases.50 This result is due to the best affinity of the hybrid ZIF with the polymer provided by the blm presence in its structure (see N2 adsorption in Fig. S6) and the reduction of the effective pore diameter, allowing a better $\mathrm{CO} 2$ discrimination by the sieving process, and thus enhancing the membrane selectivity. The increase in $\mathrm{H} 2$ permeability may be related to the larger selfdiffusion coefficient that the hybrid possesses according to the simulation results (Table S6). It can also be seen how the ZIF-7 membrane performance was worse than that of ZIF-8. This proves that the amount of blm in the hybrid material must be low $10 \% \mathrm{~mol}$ according to $\mathrm{GC} / \mathrm{MS}$ analysis) to enable the improvement in the gas separation performance, minimizing 
the $\mathrm{CO} 2$ adsorption. A MMM containing a physical mixture of ZIF-7 and ZIF-8 (10 wt\% each) was also tested to compare the results with those of ZIF-7/8 hybrid core-shell MMMs. This membrane showed an inferior separation performance as well: $\mathrm{H} 2$ permeability of 277.4 Barrer and a $\mathrm{H} 2 / \mathrm{CO} 2$ selectivity of 7.0. Finally, the $\mathrm{PBI} M M M$ with the highest loading of hybrid $\mathrm{ZIF}, 32 \mathrm{wt} \%$, was tested at a higher temperature $\left(250^{\circ} \mathrm{C}\right)$, showing values of 1921 Barrer of $\mathrm{H} 2$ and a $\mathrm{H} 2 / \mathrm{CO} 2$ selectivity of 11.8 .
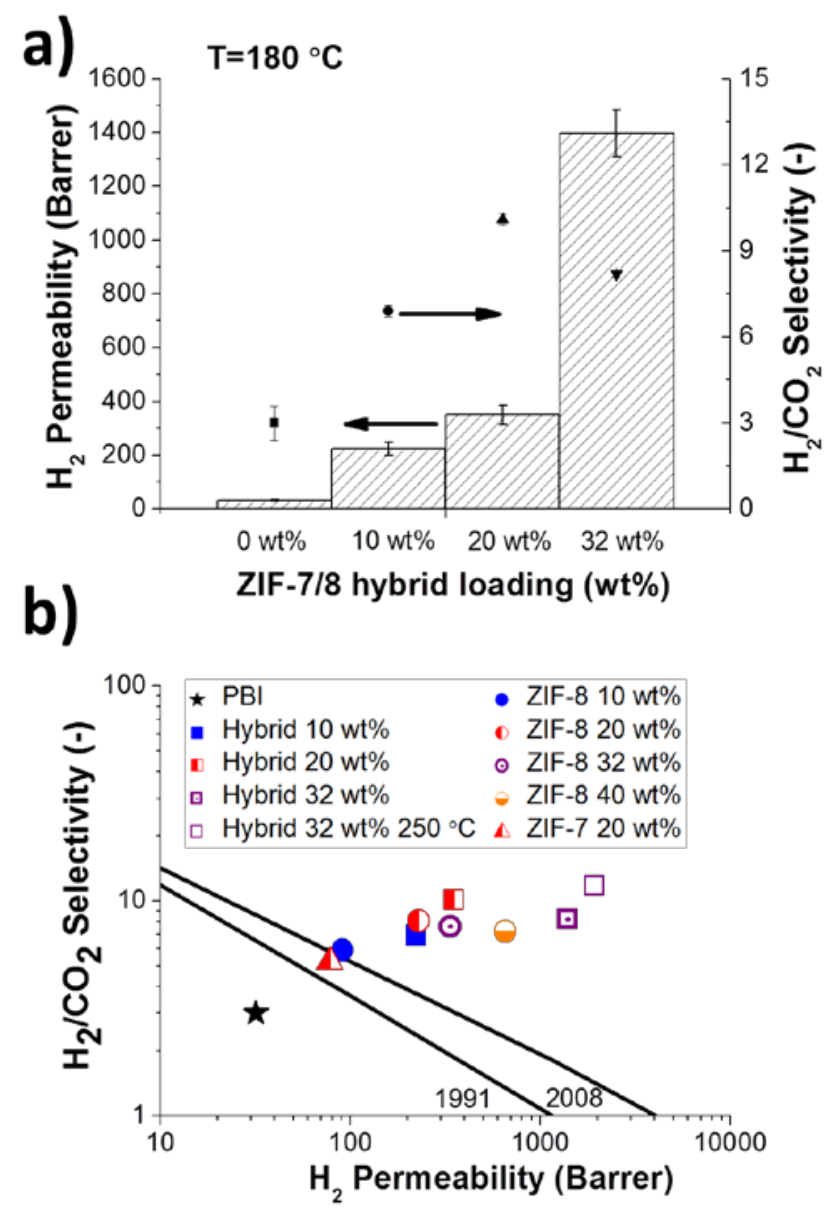

Fig. 5. Mixed gas separation performance at $180{ }^{\circ} \mathrm{C}$ of neat $\mathrm{PBI}$ and $\mathrm{PBI}$ MMMs containing the hybrid ZIF-7/8 at loadings of 10, 20 and 32 wt\% (a) and gas separation performance at 180 and $250{ }^{\circ} \mathrm{C}$ of PBI MMMs of ZIF-8, ZIF-7 and ZIF-7/8 hybrid ( $10 \% \mathrm{blm}$ ) with loadings up to $40 \mathrm{wt} \%$. Continuous lines correspond to the Robeson's upper bounds ${ }^{[50]}$ (b).

\section{4- Conclusions}

The post-synthetic modification of ZIF-8 to ZIF-7 has been successfully adjusted to the shrinking core model. This kinetic model let define with high accuracy the reaction conditions to obtain a great variety of hybrid frameworks with different compositions. Among them, hybrid ZIFs containing 10 and $44 \% \mathrm{~mol}$ of blm with sod topology were characterized in depth. The particles are envisioned as ZIF-8-ZIF-7 core-shells with a wide hybridized interphase. XRD patterns revealed a linear combination of ZIF-7 and ZIF-8 diffractograms, while TGA analyses showed a combined curve with single onset temperatures. The hybrid framework also showed 
a decrease in the N2 adsorption capacity due to the presence of blm in its structure, with a BET reduction of about $20 \%$ of the original value for $\mathrm{ZIF}-8$, and an increase in $\mathrm{CO} 2$ adsorption that was corroborated by molecular simulation results. The use of the ZIF-7/8 hybrid as a filler in $\mathrm{PBI}$ membranes clearly enhanced the gas separation performance for $\mathrm{CO} 2$ pre-combustion capture ( $\mathrm{H} 2 / \mathrm{CO} 2$ mixtures) at high temperatures due to: (a) the better filler-polymer compatibility provided by benzimidazole ligand from ZIF-7, acting as shell, and (b) the reduced $\mathrm{CO} 2$ adsorption and high $\mathrm{H} 2$ diffusivity of ZIF-8, acting as core. The concepts of core-shell and reaction monitoring may be extended to other MOFs and different applications, so that the frameworks can modify their properties to enhance their performance.

\section{Acknowledgements}

The research leading to these results has received funding from the European Union Seventh Framework Programme (FP7/2007-2013) under grant agreement $n^{\circ} 608490$, project M4CO2. In addition, financial support from the Spanish MINECO and FEDER (MAT2016-77290-R and CTP2016-80206-P), the Aragón Government (T05) and the ESF is gratefully acknowledged. J. SL. thanks Spanish Education Ministry Program FPU2014 his PhD grant. S. R. G. Balestra also thanks the Spanish MINECO for his predoctoral fellowship (BES-2014-067825 from CTQ201348396-P). All the microscopy work was done in the Laboratorio de Microscopías Avanzadas at the Instituto de Nanociencia de Aragón (LMA-INA). Finally, the authors would like to acknowledge the use of the Servicio General de Apoyo a la Investigación-SAI, Universidad de Zaragoza.

\section{Conflicts of interest}

There are no conflicts to declare.

\section{Notes and references}

1 G. Ferey and C. Serre, Chem. Soc. Rev., 2009, 38, 1380-1399.

2 B. Zornoza, C. Tellez, J. Coronas, J. Gascon and F. Kapteijn, Microporous Mesoporous Mater., 2013, 166, 67-78.

3 H. B. T. Jeazet, C. Staudt and C. Janiak, Dalton Trans., 2012, 41, 14003-14027.

4 P. Horcajada, C. Serre, M. Vallet-Regí, M. Sebban, F. Taulelle and G. Férey, Angew. Chem., 2006, 118, 6120-6124.

5 H. Jiang, T. Akita, T. Ishida, M. Haruta and Q. Xu, J. Am. Chem. Soc., 2011, 133, 1304-1306.

6 J. Gascon, U. Aktay, M. D. Hernandez-Alonso, G. P. M. van Klink and F. Kapteijn, J. Catal., 2009, 261, 75-87.

7 K. S. Park, Z. N. Ni, A. P. Côte, J. Y. Choi, R. Huang, F. J. Uribe-Romo, H. K. Chae, M. O'Keeffe and O. M. Yaghi, PNAS, 2006, 103, 10186-10191.

8 X. Huang, Y. Lin, J. Zhang and X. Chen, Angew.Chem., 2006, 118, 1587-1589. 
9 B. Chen, Z. Yang, Y. Zhu and Y. Xia, J. Mater. Chem. A, 2014, 2, 16811-16831.

10 F. Rashidi, C. R. Blad, C. W. Jones and S. Nair, AIChE J., 2016, 62, 525-537.

11 J. A. Thompson, C. R. Blad, N. A. Brunelli, M. E. Lydon, R. P. Lively, C. W. Jones and S. Nair, Chem. Mater., 2012, 24, 1930-1936.

12 C. Zhang, Y. Xiao, D. Liu, Q. Yang and C. Zhong, Chem. Commun., 2013, 49, 600-602.

13 R. Banerjee, A. Phan, B. Wang, C. Knobler, H. Furukawa, M. O'Keeffe and O. M. Yaghi, Science, 2008, 319, 939-943.

14 R. Banerjee, H. Furukawa, D. Britt, C. Knobler, M. O’Keeffe and O. M. Yaghi, J. Am. Chem. Soc., 2009, 131, 3875-3877.

15 N. T. Nguyen, H. Furukawa, F. Gándara, H. T. Nguyen, K. E. Cordova and O. M. Yaghi, Angew. Chem. Int .Ed., 2014, 53, 10645-10648.

16 S. M. Cohen, Chem. Rev., 2011, 112, 970-1000.

17 A. Huang and J. Caro, Angew. Chem. Int. Ed., 2011, 50, 4979-4982.

18 J. Canivet, S. Aguado, C. Daniel and D. Farrusseng, ChemCatChem, 2011, 3, 675-678.

19 X. Liu, Y. Li, Y. Ban, Y. Peng, H. Jin, H. Bux, L. Xu, J. Caro and W. Yang, Chem.Commun., 2013, 49, 9140-9142.

20 K. C. Jayachandrababu, D. S. Sholl and S. Nair, J. Am. Chem. Soc., 2017, 139, 5906-5915.

21 S. Choi, J. Coronas, Z. Lai, D. Yust, F. Onorato and M. Tsapatsis, J. Membr. Sci., 2008, 316, 145-152.

22 T. Yang, Y. Xiao and T. Chung, Energy Environ. Sci., 2011, 4, 4171-4180.

23 L. Li, Y. Jianfeng, X. Wang, Y. Chen and H. Wang, J. Appl. Polym. Sci., 2014, 131, 41056

24 X. Li, R. P. Singh, K. W. Dudeck, K. A. Berchtold and B. C. Benicewicz, J. Membr. Sci., 2014, $461,59-68$.

25 S. Kumbharkar, Y. Liu and K. Li, J. Membr. Sci., 2011, 375, 231-240.

26 B. P. Biswal, A. Bhaskar, R. Banerjee and U. K. Kharul, Nanoscale, 2015, 7, 7291-7298.

27 T. Yang, G. M. Shi and T. Chung, Adv. Energy Mater., 2012, 2, 1358-1367.

28 T. Yang and T. Chung, Int. J. Hydrogen Energy, 2013, 38, 229-239.

29 A. Bhaskar, R. Banerjee and U. Kharul, J. Mater.Chem. A, 2014, 2, 12962-12967.

30 J. Sánchez-Laínez, B. Zornoza, C. Téllez and J. Coronas, J. Mater.Chem. A, 2016, 4, $14334-$ 14341.

31 T. Yang and T. Chung, J. Mater.Chem. A, 2013, 1, 6081-6090. 
32 N. Liédana, A. Galve, C. Rubio, C. Téllez and J. Coronas, ACS Appl. Mater. Interfaces, 2012, 4, 5016-5021.

33 Y.-S. Li, F.-Y. Liang, H. Bux, A. Feldhoff, W.-S. Yang, J. Caro, Andgew. Chem. Int. Ed., 2010, $548-551$.

34 D. Dubbeldam, S. Calero, D. E. Ellis and R. Q. Snurr, Mol. Simul., 2016, 42, 81-101.

35 J. J. G. Sevillano, S. Calero, C. O. Ania, J. B. Parra, F. Kapteijn, J. Gascon and S. Hamad, J. Phys. Chem. C, 2012, 117, 466-471.

36 A. K. Rappé, C. J. Casewit, K. Colwell, W. Goddard lii and W. Skiff, J. Am. Chem. Soc., 1992, 114, 10024-10035.

37 S. L. Mayo, B. D. Olafson and W. A. Goddard, J. Phys. Chem., 1990, 94, 8897-8909.

38 D. Dubbeldam and R. Snurr, Mol. Simul., 2007, 33, 305-325.

39 X. Wu, J. Huang, W. Cai and M. Jaroniec, RSC Advances, 2014, 4, 16503-16511.

40 S. Yagi and D. Kunii, 1955, 5, 231-244.

41 B. Zheng, L. Wang, L. Du, Y. Pan, Z. Lai, K. Huang and H. Du, Mater.Horiz., 2016, 3, 355-361.

42 S. Aguado, G. Bergeret, M. P. Titus, V. Moizan, C. Nieto-Draghi, N. Bats and D. Farrusseng, New J. Chem., 2011, 35, 546-550.

43 W. Morris, N. He, K. G. Ray, P. Klonowski, F. Hiroyasu, I. N. Daniels, Y. A. Houndonougbo, M. Asta, O. M. Yaghi and B. B. Laird, J. Phys. Chem., 2012, 116, 24084-24090.

44 U. Böhme, C. Paula, V. Reddy Marthala, J. Caro and M. Hartmann, Chem. Ing.Technik, 2013, $85,1707-1713$.

45 A. S. Cattaneo, D. C. Villa, S. Angioni, C. Ferrara, E. Quartarone and P. Mustarelli, J. Phys. Chem. C, 2015, 119, 18935-18944.

46 J. Grobelny, D. M. Rice, F. E. Karasz and W. J. MacKnight, Macromolecules, 1990, 23, 2139 2144.

47 R. M. Claramunt, C. López, I. Alkorta, J. Elguero, R. Yang and S. Schulman, Magn. Reson. Chem., 2004, 42, 712-714.

48 B. Zornoza, S. Irusta, C. Téllez and J. Coronas, Langmuir, 2009, 25, 5903-5909.

49 R. Mahajan, R. Burns, M. Schaeffer and W. J. Koros, J. Appl. Polym. Sci., 2002, 86, 881-890.

50 L. M. Robeson, J. Membr. Sci., 2008, 320, 390-400. 